\title{
Lesson of the month 1: Artery of Percheron occlusion - an uncommon cause of coma in a middle-aged man
}

\author{
Authors: James Bailey ${ }^{A}$ and Kayvan Khadjooi ${ }^{B}$
}

\begin{abstract}
Bilateral paramedian thalamic infarction resulting from artery of Percheron occlusion presents with a distinct clinical syndrome comprising impaired consciousness, often with vertical gaze palsy and memory impairment. This uncommon anatomical variant arises as a single artery supplying both paramedian thalami. Early recognition can be challenging in the obtunded patient, where the differential diagnosis is broad. The acute physician should consider this diagnosis in a patient presenting with unexplained coma so that emergent treatments such as thrombolysis can be employed. Early imaging with computerised tomography can often be normal; therefore the use of magnetic resonance imaging is essential in confirming the diagnosis.
\end{abstract}

\section{Case presentation}

A previously fit and well 47-year-old man presented to the emergency department after being found unresponsive by his wife in bed in the morning. He had been well the previous evening, however he had taken a combination of opioid and antiinflammatory medications for shoulder pain earlier in the day. An ambulance was called and he was found to be profoundly drowsy with a Glasgow Coma Scale (GCS) of 3/15 with pupil diameter $1 \mathrm{~mm}$ bilaterally. He was treated empirically for opioid toxicity, in view of his use of opioid-based analgesics and pinpoint pupils, and an improvement in his level of alertness was noted.

On arrival to the emergency department his blood pressure was $130 / 89 \mathrm{mmHg}$, heart rate 81 beats/min in sinus rhythm, and his respiratory rate $20 / \mathrm{min}$. On neurological exam, his GCS was 15 . His pupils were $2 \mathrm{~mm}$ in diameter bilaterally and reactive to light. There was no focal neurological deficit noted. Rash and evidence of meningeal irritation was absent. Cardiorespiratory examination was unremarkable. In the initial course of his admission he demonstrated fluctuations in his level of alertness ranging from deep coma to mild somnolence. $\mathrm{He}$ and his wife also reported problems with short-term memory since the onset of his symptoms.

Authors: ${ }^{\text {A }}$ specialty trainee in stroke medicine, Addenbrooke's Hospital, Cambridge University Hospital NHS Foundation Trust,

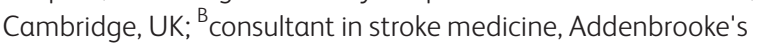
Hospital, Cambridge University Hospital NHS Foundation Trust, Cambridge, UK

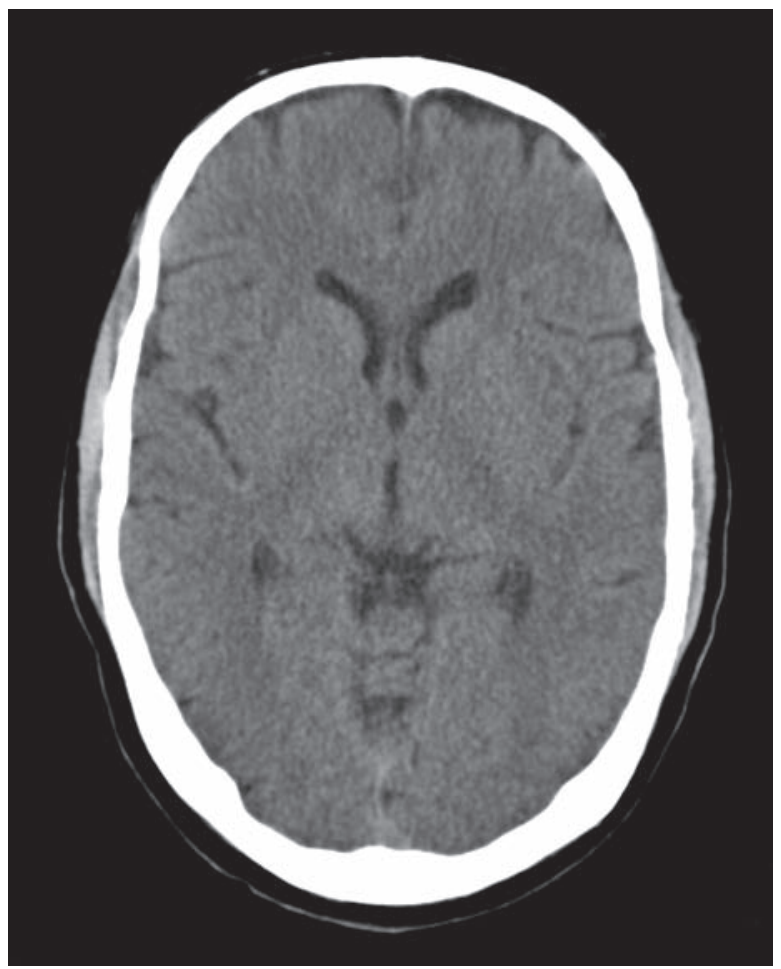

Fig. 1. Non-contrast computerised tomography head on admission.

\section{Investigations}

His initial investigations were as follows: $\mathrm{Na} 141 \mathrm{mEq} / \mathrm{L}, \mathrm{K}$ $4.1 \mathrm{mEq} / \mathrm{L}$, urea $4.5 \mathrm{mmol} / \mathrm{L}$, creatinine $64 \mu \mathrm{mol} / \mathrm{L}$, pH 7.36, C-reactive protein undetectable, glucose $5.9 \mathrm{mmol} / \mathrm{L}$, bilirubin $6 \mathrm{mmol} / \mathrm{L}$, alanine transaminase $36 \mathrm{IU} / \mathrm{L}(0-33 \mu \mathrm{mol} / \mathrm{L})$, alkaline phosphatase $60 \mathrm{IU} / \mathrm{L}$, white blood cells $11.0 \times 10^{9} / \mathrm{L}$ $\left(4.0-11.0 \times 10^{9} / \mathrm{L}\right)$, neutrophils $8.4 \times 10^{9} / \mathrm{L}\left(2.0-7.5 \times 10^{9} / \mathrm{L}\right)$, haemoglobin $153 \mathrm{~g} / \mathrm{L}$ and platelets $282 \times 10^{9} / \mathrm{L}$. A 12-lead electrocardiogram (ECG) showed normal sinus rhythm. Urinalysis showed no proteinuria or haematuria.

An emergent computerised tomography (CT) scan of his head was arranged, which was normal (Fig 1). Treatment with naloxone was repeated in view of an apparent response on initial assessment, but his symptoms persisted so he proceeded to magnetic resonance imaging (MRI). This revealed bilateral paramedian thalamic infarction in keeping with artery of 


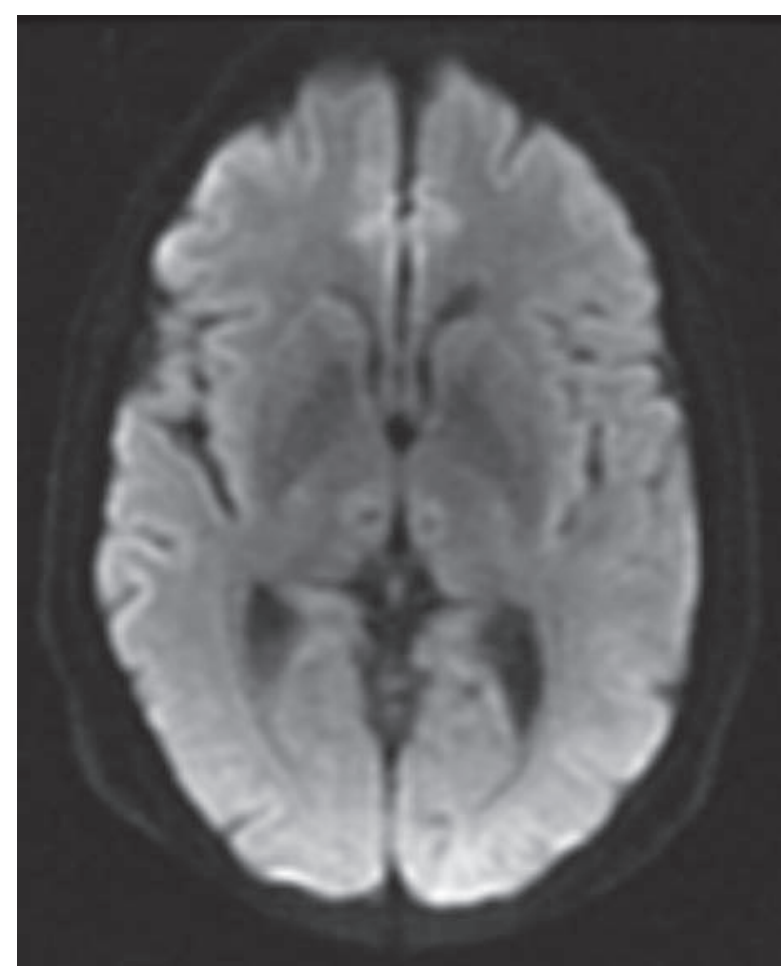

Fig. 2. Magnetic resonance imaging head with diffusion weighted imaging.

Percheron (AOP) occlusion, evidenced by restricted diffusion with areas of petechial haemorrhage within the infarcts (Fig 2). CT angiography showed normal carotids and vertebral arteries bilaterally, but a thin basilar artery as a result of bilateral fetaltype posterior communicating arteries.

He was commenced on clopidogrel and simvastatin and discharged home the following day. On neurocognitive testing he displayed a marked deficit with delayed recall. Further investigations to elucidate the cause of his stroke were unrevealing, including a normal 24-hour ECG and transthoracic echocardiogram. His total cholesterol was $4.0 \mathrm{mmol} / \mathrm{L}$ and fasting glucose was $4.8 \mathrm{mmol} / \mathrm{L}$. A thrombophilia screen including lupus anticoagulant and anti-cardiolipin antibodies was negative.

At three-month follow-up he had continued to make a good recovery with only a minor degree of orthostatic vertigo, but no persistent memory deficit and was planning his return to work in the near future.

\section{Discussion}

Approximately $5 \%$ of presentations to the emergency departments are with alerted mental status, of which $63 \%$ are admitted to hospital. ${ }^{1}$ In the patient presenting to hospital with coma, a thorough and systematic approach is required to look for a reversible cause. ${ }^{2}$ Acute strokes comprise between 6 and $54 \%$ of hospital admissions with coma, and a high index of suspicion is required particularly as CT may be normal in the early stages after presentation as was the case in our patient. ${ }^{3,4}$

This case demonstrates the challenges in diagnosing AOP occlusion and subsequent paramedian thalamic infarction in a patient presenting with reduced consciousness, which can closely mimic other causes of coma. In one review of patients with AOP occlusion, 32 out of 120 patients were noted to have pupillary abnormalities, of which 12 had miosis, which can lead to suspicion of opioid toxicity. ${ }^{4}$ Similarly, other posterior circulation stroke syndromes resulting from ischaemia or haemorrhage can also present with altered consciousness and pupil abnormalities, usually associated with other focal neurological deficits. ${ }^{5}$ In another smaller series, 2 out of 6 patients displayed a pattern of episodic coma lasting 30 minutes at a time in the first days after presentation as was demonstrated by our patient. ${ }^{6}$ In patients presenting with unexplained, non-traumatic coma, consideration should be given to early imaging with CT and CT angiography to exclude haemorrhage and basilar artery thrombosis respectively. Early MRI, where available, has far superior sensitivity to CT and should be employed to confirm diagnosis. ${ }^{2,5}$ MR angiography has the additional benefit of being able to distinguish between two important causes of bilateral paramedian thalamic infarction; top of basilar thrombosis and deep cerebral vein thrombosis and is superior to CT in identifying ischaemic lesions, particularly in the acute phase. ${ }^{2,7}$

\section{Conclusion}

AOP occlusion is an uncommon cause of coma presenting in elderly and middle-aged individuals. The patient may have a source of cardioembolism or classical cardiovascular risk factors. Reduced consciousness can range from deep coma to hypersomnolence. Opthamological signs and neuropsychological deficits may also be evident. Despite investigation the cause remains undetermined in the majority of cases. Overall the prognosis is good with low mortality and often rapid reversal of the coma, necessitating early diagnosis and optimal supportive care. ${ }^{4}$

Reaching the correct diagnosis in patients presenting with non-traumatic coma can be challenging, but incorrect diagnosis can result in delays in appropriate management and potential for harm due to employment of unnecessary treatments and ongoing symptoms. This case highlights the difficulty in recognising a stroke syndrome presenting as isolated reduced consciousness and the importance of revisiting a diagnosis when a patient fails to respond to initial treatment.

\section{References}

1 Kanich W, Brady WJ, Huff JS et al. Altered mental status: evaluation and etiology in the ED. Am J Emerg Med 2002;20:613-7.

2 Edlow JA, Rabinstein A, Traub SJ, Wijdicks EF. Diagnosis of reversible causes of coma. Lancet 2014;384:2064-76.

3 Horsting MW, Franken MD, Meulenbelt J, van Klei WA, de Lange DW. The etiology and outcome of non-traumatic coma in critical care: a systematic review. BMC Anesthesiol 2015;15:65.

4 Zappella N, Merceron S, Nifle C et al. Artery of Percheron infarction as an unusual cause of coma: three cases and literature review. Neurocrit Care 2014;20:494-501.

5 Merwick A, Werring D. Posterior circulation ischaemic stroke. BMJ 2014;348:g3175.

6 Reilly M, Connolly S, Stack J, Martin EA, Hutchinson M. Bilateral paramedian thalamic infarction: a distinct but poorly recognized stroke syndrome. Q J Med 1992;82:63-70.

7 Linn J, Hoffmann LA, Danek A, Bruckmann H. Differential diagnosis of bilateral thalamic lesions. Rofo 2007;179:234-45.

Address for correspondence: Dr ] Bailey, EAU Office (S612), Ipswich Hospital NHS Trust, Heath Road, Ipswich IP4 5PD, UK. Email: james.bailey5@nhs.net 